\title{
Basic fibroblast growth factor treatment for non-steroidal anti-inflammatory drug associated gastric ulceration
}

\author{
M A Hull, D J E Cullen, N Hudson, C J Hawkey
}

\begin{abstract}
An open, pilot study tested the safety and efficacy of an acid stable form of the angiogenic protein basic fibroblast growth factor (bFGF) for healing of non-steroidal anti-inflammatory drug (NSAID) associated gastric ulcers in five patients with nine gastric ulcers that were resistant to conventional treatment (4) or had relapsed (5). Oral bFGF treatment for four weeks was safe and well tolerated. There was no evidence of systemic absorption. After four weeks, four ulcers had healed and there was a 89 (3)\% mean (SEM) reduction in the area of the others. A controlled, comparative trial of this novel growth factor treatment is warranted.

(Gut 1995; 37: 610-612)
\end{abstract}

Keywords: basic fibroblast growth factor, TGP-580, gastric ulcer, non-steroidal anti-inflammatory drugs.

About $15 \%$ of patients taking non-steroidal anti-inflammatory drugs (NSAIDs) regularly have a gastric ulcer if endoscoped at any given time. ${ }^{1}$ Morbidity and mortality from ulcer complications such as haemorrhage and perforation are considerable. In 1991 there were 4334 recorded deaths in England and Wales attributable to peptic ulcer disease, roughly $30 \%$ of which were attributable to NSAID use. ${ }^{1}$

NSAIDs impair gastric ulcer healing ${ }^{2}$ as a result of multiple inhibitory effects on healing mechanisms; these include a reduction in epithelial cell and fibroblast proliferation and there is evidence of impaired angiogenesis (formation of capillary blood vessels) in granulation tissue at the rim of NSAID associated ulcers. ${ }^{3}$

The angiogenic protein basic fibroblast growth factor (bFGF) is found in human gastric mucosa. ${ }^{4}$ There is evidence that bFGF plays an important part in ulcer healing. In rats, a neutralising antibody against bFGF impairs gastric ulcer healing ${ }^{5}$ while exogenous bFGF treatment promotes angiogenesis and healing of gastric and duodenal ulcers. ${ }^{6}$

We have previously shown that concentrations of bFGF in patients with gastric ulceration are considerably depressed (mean 23-fold) compared with patients without ulcers. ${ }^{7}$ We therefore tested the safety and efficacy of exogenous bFGF 'replacement' treatment in a small number of patients with NSAID associated gastric ulcers. We used a derivative of human recombinant bFGF pro- duced by site directed mutagenesis to achieve greater stability at acidic pH (bFGF CS23; TGP-580, Takeda Chemical Industries, Osaka, Japan). ${ }^{8}$ TGP-580 has angiogenic activity at normal gastric $\mathrm{pH}$ and has previously been given orally to eight healthy volunteers with no adverse events reported (Folkman et al unpublished data). As bFGF promotes its own synthesis in cell culture, we also tested whether treatment with TGP-580 would raise the low endogenous mucosal bFGF concentrations of patients treated to those seen in ulcer free subjects.

\section{Methods}

Patients were suitable for inclusion into the study if they required continuing NSAID treatment for clinical reasons and had one or more gastric ulcers at endoscopy. An ulcer was defined as a break in the gastric mucosa with ulcer slough and unequivocal depth. Other ulcer healing treatment including antacids was not permitted during the study.

TGP- 580 was stored at $-20^{\circ} \mathrm{C}$ until given to patients at which time TGP-580 was stored in the home refrigerator until ingestion. Patients received $0.1 \mathrm{mg}$ TGP-580 in $10 \mathrm{ml}$ citrate buffer, twice daily, after meals for 28 days. One gram of sodium bicarbonate was given simultaneously with TGP-580 to increase gastric $\mathrm{pH}$ transiently and enhance TGP-580 stability.

During the study patients filled in a symptom diary and were asked to report any new symptoms. The first dose of TGP-580 was taken under medical supervision with haemodynamic monitoring for two hours. Blood was taken for serum TGP-580 measurement and routine haematology and biochemistry (full blood count and differential, urea and electrolytes, liver function tests, glucose and amylase) one hour after initial TGP-580 ingestion and before each endoscopy.

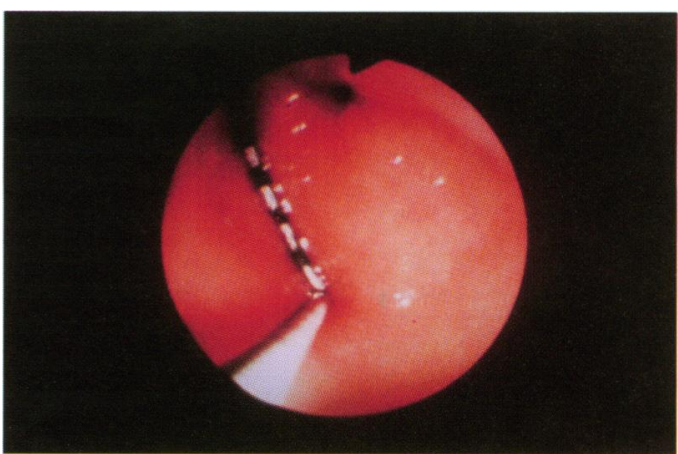

Figure 1: The endoscopic ruler measuring device adjacent to antral mucosa. Each gradation represents $2 \mathrm{~mm}$. 


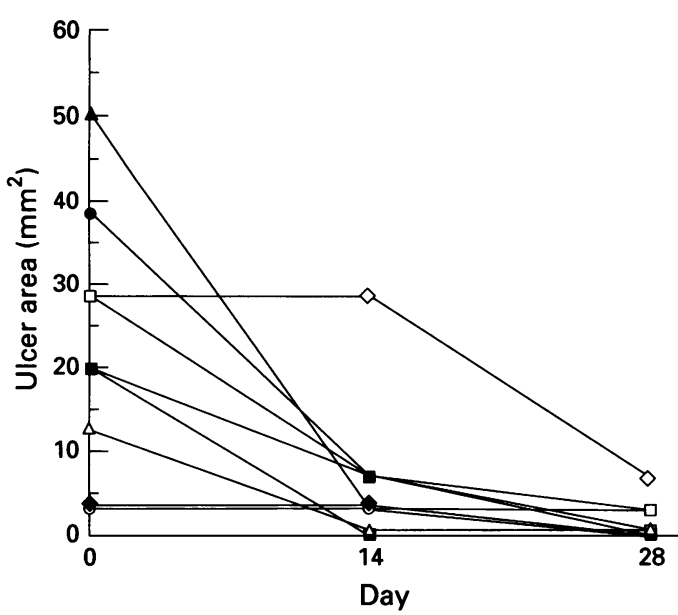

Figure 2: Time course of reduction in area of NSAID associated gastric ulcers during TGP-580 treatment. Each line represents an individual ulcer.

Each patient underwent endoscopy at entry (day 0 ), at 14 days, and at the end of the study (day 28). All endoscopies were performed by one operator $(\mathrm{MH})$. During endoscopy, the position of each gastric ulcer was noted and the
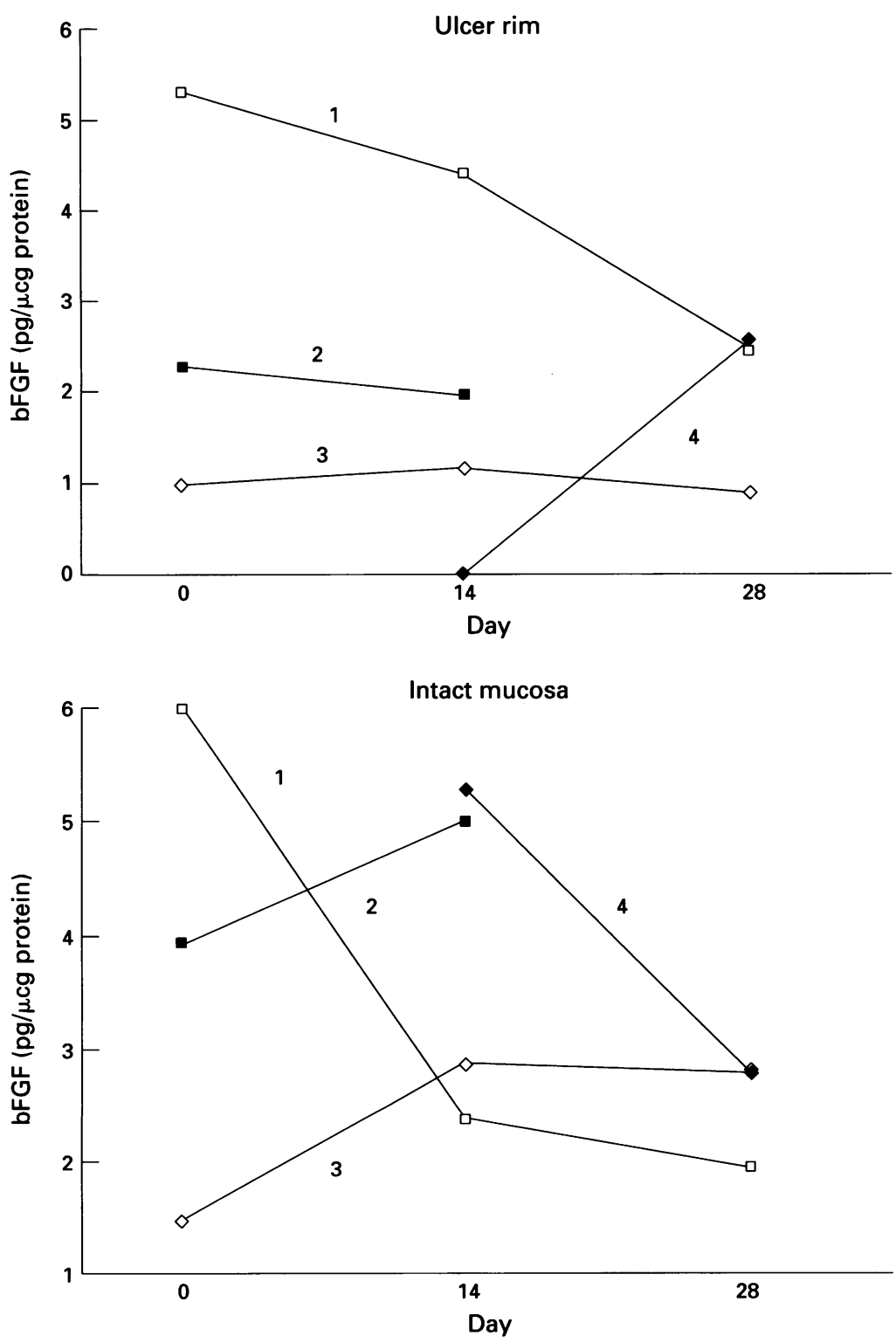

Figure 3: Mucosal bFGF concentrations at the ulcer rim and in intact mucosa during TGP-580 treatment. Each line represents individual patients. diameter of ulcer slough measured using a custom-made ruler device placed at the ulcer surface through the endoscope biopsy channel (Fig 1). The use of the ruler device was validated as described previously. ${ }^{9}$ Ulcer area was calculated from the diameter $\left(\pi r^{2}\right)$. Erosions and petechiae in the body, antrum, and duodenum were also counted. Two biopsy specimens were taken from the rim of one ulcer and neighbouring intact mucosa whenever possible. Specimens were then homogenised in TRIS-saline, centrifuged, and the supernatant collected. Mucosal immunoreactive bFGF and TGP-580 concentrations were measured using specific enzyme linked immunosorbent assays ${ }^{10}$ and related to total protein content.

The study was approved by the University Hospital Ethics Committee.

\section{Results}

Five patients were enrolled into the study (four female, one male; mean (SEM) age 56 (4) years). All were taking NSAIDs regularly for rheumatoid arthritis (4) or headaches/arthritis (1). Two patients had four gastric ulcers that were resistant to continuing $\mathrm{H}_{2}$ receptor antagonist treatment and three patients had five gastric ulcers that developed during endoscopic surveillance. Two patients were Helicobacter pylori positive by urease test. Only one patient was a smoker.

The ruler device was validated as follows; video recordings of 21 NSAID associated ulcers and erosions were made. The diameter of lesions was measured independently by six endoscopists. Data were analysed by two way analysis of variance. The ruler distinguished significantly between different sized lesions $(p<0.0001)$. However, there was also significant variation between endoscopists $(p=0.01)$ and so the coefficient of interobserver variation was relatively high $(41 \%)$.

In all, nine gastric ulcers were present in the five patients ( 6 antrum, 3 body; mean (SEM) area $22.6(5 \cdot 2) \mathrm{mm}^{2}$ ). One gastric ulcer (initial diameter $5 \mathrm{~mm}$ ) had healed completely by 14 days and three more by 28 days. Mean initial area of gastric ulcers remaining unhealed at four weeks was $27 \cdot 8(6 \cdot 3) \mathrm{mm}^{2}$ compared with $16 \cdot 1(8 \cdot 4) \mathrm{mm}^{2}$ for gastric ulcers that healed (difference not significant). There was a 89 (3)\% (mean (SEM) reduction in area of the five ulcers that remained unhealed at four weeks. Figure 2 shows the time course of gastric ulcer healing. No new ulcers were detected during the study. There were no significant changes in the number of erosions or petechiae during the study.

Paired mucosal biopsy samples from ulcer rim and intact mucosa were obtained on days 0 $(n=3), 14(n=4)$, and $28(n=3)$. There was no obvious increase in mucosal bFGF, which remained grossly depressed during TGP-580 treatment (Fig 3). TGP-580 itself was not detected in gastric mucosa from either the ulcer rim or intact mucosa.

No TGP-580 was detected in serum at any time during the study nor were there any 
changes in biochemical or haematological parameters measured. No adverse events attributable to TGP-580 or sodium bicarbonate were noted. However, one patient described worse dyspepsia during the study compared with prior treatment with $\mathrm{H}_{2}$ receptor antagonists.

\section{Discussion}

This open, pilot study has shown that oral bFGF treatment for four weeks is safe and well tolerated and has potential as a new treatment for NSAID associated gastric ulceration. Measurement of ulcer size with the ruler device showed accurately a reduction in area of unhealed ulcers. As only one endoscopist was used we eliminated interobserver variation using the ruler. We do not believe that the dose of sodium bicarbonate given was sufficient to effect ulcer healing in itself (neutralising activity $20 \mathrm{mEq} /$ day). Previous studies have shown that $120 \mathrm{mEq}$ neutralising activity per day is required for healing of non-NSAID gastric ulcers. ${ }^{11}$ The lack of evidence of systemic absorption of bFGF supports the view that the mode of action is largely or exclusively topical. We were unable to detect TGP-580 in ulcers in this study possibly because of the long interval between the last dose and endoscopy. TGP-580 did not seem to induce an increase in endogenous bFGF, which remained considerably depressed compared with values we have found in non-ulcer patients. ${ }^{7}$

The lack of prior toxicological data did not allow the study to proceed longer than four weeks. A controlled, comparative study of
TGP-580 for NSAID associated gastric ulcer healing over a longer period would help to define its therapeutic potential. As reduced mucosal bFGF concentrations are associated with gastric ulcers, exogenous bFGF may be thought of as treatment of bFGF 'deficiency' and could become treatment for healing or prophylaxis against gastric ulceration, or both.

This work has previously been presented in abstract form at the American Gastroenterological Association meeting, 15-18 May 1994. ${ }^{7}$

1 Hawkey CJ. Non-steroidal anti-inflammatory drugs and peptic ulcers. Facts and figures multiply, but do they add up? BMF 1990; 300: 278-84.

2 Lancaster-Smith MJ, Jaderberg MR, Jackson DA. Ranitidine in the treatment of non-steroidal anti-inflammatory drug-associated gastric and duodenal ulcers. Gut 1991; 32: 252-6.

3 Hudson N, Balsitis M, Everitt S, Hawkey CJ. Angiogenesis in gastric ulcers; impaired in patients taking non-steroidal anti-inflammatory drugs. Gut 1995; 37: 191-4.

4 Hull MA, Morrell K, Hawkey CJ, Jenkins DJ. Localization of basic fibroblast growth factor in gastric ulcers. Gut 1994; 35 (suppl 5): S49.

5 Satoh H, Shino A, Sato F, Inatomi N, Kozai Y, Kato K, et al. Role of endogenous and exogenous bFGF in the healing of gastric ulcers in the rat. Gastroenterology 1992;102: A159.

6 Szabo S, Folkman J, Vattey P, Morales RE, Pinkus GS, Kato $K$. Accelerated healing of duodenal ulcers by oral administration of a mutein of basic fibroblast growth factor in rats. Gastroenterology 1994; 106: 1106-11.

7 Hull MA, Cullen DJE, Hawkey CJ. Basic fibroblast growth factor in gastric ulceration: mucosal levels and therapeutic

8 Seno M, Sasada R, Iwane M, Sudo K, Kurokawa T, Ito K, et al. Stabilizing basic fibroblast growth factor using protein engineering. Biochem Biophys Res Commun 1988; 151: 701-8.

9 Hudson N, Everitt S, Hawkey CJ. Interobserver variation in assessment of gastroduodenal lesions associated with nonsteroidal anti-inflammatory drugs. Gut 1994; 35: 1030-2.

10 Watanabe H, Hori A, Seno M, Kozai Y, Igarashi K, Ichimori $\mathrm{Y}$, et al. A sensitive enzyme immunoassay for human basic fibroblast growth factor. Biochem Biophys Res Commun 1991; 175: 229-35.

11 Rydning A, Weberg R, Lange O, Berstad A. Healing of benign gastric ulcer with low-dose antacids and fiber diet. Gastroenterology 1986; 91: 56-61. 